\title{
APPROXIMATE RECONSTRUCTION OF BANDLIMITED FUNCTIONS FOR THE INTEGRATE AND FIRE SAMPLER
}

\author{
HANS G. FEICHTINGER, JOSÉ C. PRÍNCIPE, JOSÉ LUIS ROMERO, \\ ALEXANDER SINGH ALVARADO, AND GINO ANGELO VELASCO
}

\begin{abstract}
In this paper we study the reconstruction of a bandlimited signal from samples generated by the integrate and fire model. This sampler allows us to trade complexity in the reconstruction algorithms for simple hardware implementations, and is specially convenient in situations where the sampling device is limited in terms of power, area and bandwidth.

Although perfect reconstruction for this sampler is impossible, we give a general approximate reconstruction procedure and bound the corresponding error. We also show the performance of the proposed algorithm through numerical simulations.
\end{abstract}

Keywords: integrate and fire, non-uniform sampling, bandlimited function.

\section{INTRODUCTION}

The integrate and fire (IF) model is well known in computational neuroscience as a simplified model of a neuron [8, 12, and is typically used to study the dynamics of large populations. The model consists of a leaky integrator followed by a comparator. The leak corresponds to a gradual loss of the value of the integral.

More recently, the IF model has also been considered as a sampler 4, 16, 10, 11, where the sampler output is tuned to the variation of the integral of the signal. This feature can be exploited when sampling neural recordings, for which relevant information is localized in small intervals where the signal has a high amplitude [3].

The block diagram of the sampler is presented in Figure 1. At every instant $s$, the continuous input $x(t)$ is integrated against an averaging function $u_{k, s}(t)$ and the result is compared to a positive and negative threshold. When either of these is reached, a pulse is created at time $t_{k}=s$ representing the threshold value (positive or negative), the value of the integrator is then reset and the process repeats. The

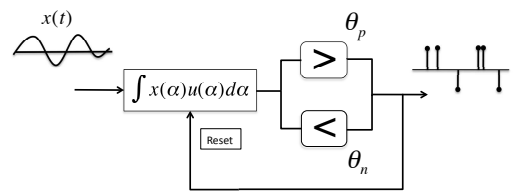

FiguRE 1. Block diagram for the BIF model.

output is a nonuniformly spaced pulse train, where each of the pulses is either 1 or -1 . The averaging function $u_{k, s}(t)$ is defined by $e^{\alpha(t-s)} \mathcal{X}_{\left[t_{k}, s\right]}$, where $\mathcal{X}_{I}$ is the characteristic function of $I$ and $\alpha>0$ is a constant that models the leakage 
of the integrator due to practical implementations. The precise firing condition determining the pulses is:

$$
\pm \theta=\int_{t_{k}}^{t_{k+1}} f(t) e^{\frac{-\left(t_{k+1}-t\right)}{\alpha}} d t=:\left\langle f, u_{k}\right\rangle .
$$

The simplicity of the sampler translates into an efficient hardware implementation which saves both power and area when compared to conventional analog-to-digital converters (ADC) 4]. These constraints are severe, in the case of wireless brain machine interfaces [13, for which the entire system has to be embedded inside the subject. Hence, the IF sampler allows us to move the complexity of the design into the reconstruction algorithm while providing a simple front end at the sampling stage.

The problem of reconstructing a signal from the IF output should be distinguished from the study of the dynamics of a population of neurons, when some stochastic assumption is made on the firing parameters [9. In this article we study the deterministic reconstruction of a bandlimited signal from the integrate and fire output. Part of the challenge of this stems from the fact that the sampling map that associates a function to its samples is non-linear. Indeed, we see from Equation (1) that the magnitude of the samples is always $\theta$. Moreover, exact reconstruction for the IF sampler is impossible since the output of the sampler does not completely determine the signal (see Example 1 below.)

In this article, we will show that it is however possible to approximately reconstruct a bandlimited signal in $L^{\infty}$ norm with an error comparable to the threshold $\theta$. Moreover, we give a concrete reconstruction procedure which is of course non-linear but, nevertheless, easy to implement. Since in many situations the IF sampler is so much more convenient to implement than conventional analog-to-digital converters, the loss of accuracy in the reconstruction is a very reasonable trade-off 4, specially if the final analysis of the reconstructed data tolerates some small error [3].

The methods considered so far [11 reconstruct the signal $f$ from the system of equations $\left\langle f, u_{k}\right\rangle= \pm \theta$ (cf. Equation (1)), thus treating the reconstruction as a (linear) average sampling problem (see [7, 1, 15, 14]). These approaches impose density restrictions on the set of sampling functions $\left\{u_{k}\right\}_{k}$ (cf. Equation (11).) Since these sampling functions depend on the signal, the density constraints on them are somehow unnatural.

The key for the reconstruction method that we develop lies in the observation that the information derived from the IF output is much richer than the mere system of equations $\left\langle f, u_{k}\right\rangle= \pm \theta$. It also contains the information that no proper subinterval $\left[t_{k}, t^{\prime}\right]$ of $\left[t_{k}, t_{k+1}\right]$ satisfies Equation (1). We will exploit this extra information to give an approximate reconstruction procedure for a general bandlimited function. Since the sampling process starts at a certain instant $t_{0}$, an additional assumption on the size of $f$ before $t_{0}$ is required in order to fully reconstruct $f$. Roughly speaking, the assumption means that the sampling scheme would not have produced any pulse before $t_{0}$.

In Section 2 we formally describe the output of the IF sampling scheme. This output depends on an initial time $t_{0}$ when the process is started and two parameters: the threshold $\theta$ and the constant $\alpha>0$ modeling the leakage on the sampler. In Section 3 we show that the IF output is always a finite sequence. Section 4 gives the approximate reconstruction procedure and Section 5 presents some numerical experiments. 


\section{The Integrate AND FIRE SAMPLING PROBLEM}

We now define precisely the integrate and fire sampling scheme. Throughout the article we will assume the following.

Assumption 1. A bandlimited function $f \in P W_{\Omega}$ and numbers $t_{0} \in \mathbb{R}, \alpha, \theta>0$ are given.

Here, $P W_{\Omega}$ is the Paley-Wiener space

$$
P W_{\Omega}:=\left\{f \in L^{2}(\mathbb{R}) \mid \operatorname{supp}(\hat{f}) \subseteq[-\Omega, \Omega]\right\},
$$

of (complex-valued) bandlimited functions and $\hat{f}(w):=\int_{\mathbb{R}} f(x) e^{-2 \pi i w x} d x$ is the Fourier transform of $f$. We call $t_{0}$ the initial time, $\alpha$ the firing parameter and $\theta$ the threshold. Using these parameters we formally define the output of the sampler. We first define recursively a finite or countable sequence $t_{0}<\ldots<t_{j} \ldots$ called the time instants. Suppose that the instants $t_{0}<\ldots<t_{j}$ have already been defined and consider the function $F_{j}:\left[t_{j},+\infty\right) \rightarrow \mathbb{C}$ given by

$$
F_{j}(t):=\int_{t_{j}}^{t} f(x) e^{\alpha(x-t)} d x
$$

Observe that $F_{j}$ is continuous and $F_{j}\left(t_{j}\right)=0$. If $\left|F_{j}(t)\right|<\theta$, for all $t \geq t_{j}$, then the process stops. If $\left|F_{j}(t)\right| \geq \theta$, for some $t \geq t_{j}$, by the continuity of $F_{j}$, we can define $t_{j+1}$ as the minimum number satisfying the equation

$$
\left|\int_{t_{j}}^{t_{j+1}} f(x) e^{\alpha\left(x-t_{j+1}\right)} d x\right|=\theta .
$$

Clearly, in this case $t_{j+1}>t_{j}$.

We have defined a finite or countable sequence of points $t_{0}<\ldots<t_{j} \ldots$. We will prove in Proposition 1 that this sequence is in fact finite. Let us assume the time instants $\left\{t_{0}, \ldots, t_{n}\right\}$ and define the samples $\left\{q_{1}, \ldots, q_{n}\right\}$ by,

$$
q_{j}:=\int_{t_{j-1}}^{t_{j}} f(x) e^{\alpha\left(x-t_{j}\right)} d x, \quad(1 \leq j \leq n) .
$$

Observe that, by the definition of the time intervals, $\left|q_{j}\right|=\theta$.

The output of the sampler is formally given by the time instants $\left\{t_{0}, \ldots, t_{n}\right\}$ and the numbers $\left\{q_{1}, \ldots, q_{n}\right\}$. We say that this output has been produced by the integrate and fire. The succeeding results apply generally to complex-valued functions, but in the case of the application that motivated this sampling scheme, the signal is taken to be real-valued, and the output of the sampler is encoded as a train of impulses, where only the sign of the samples $q_{j}$ is stored.

\section{Some ReMARKS ON THE IF OUTPUT}

First we note that bandlimited functions are not completely determined by the output of the IF sampler.

Example 1. There are non-zero bandlimited signals that will never produce an output from the sampler. Take for instance $f_{\theta}(x)=\frac{\theta \sin ^{2}(\pi x)}{2 \pi^{2} x^{2}}$. Since $\hat{f}_{\theta}(\omega)=$ $\frac{\theta}{2} \max \{1-|\omega|, 0\}, f_{\theta}$ is bandlimited. We have for any $t_{0} \in \mathbb{R},\left|\int_{t_{0}}^{t} f_{\theta}(x) e^{\alpha(x-t)} d x\right| \leq$ $\int_{t_{0}}^{t}\left|\frac{\theta \sin ^{2}(\pi x)}{2 \pi^{2} x^{2}}\right| d x \leq \frac{\theta}{2} \int_{\mathbb{R}} \frac{\sin ^{2}(\pi x)}{\pi^{2} x^{2}} d x=\frac{\theta}{2}<\theta$, for all $t \geq t_{0}$. 
We now prove that the set of time instants produced by the IF sampler is indeed finite and give some bounds on its distribution. To this end we introduce some auxiliary functions that will be used throughout the remainder of the article.

Consider the function $g: \mathbb{R} \rightarrow \mathbb{R}$ given by $g(x)=e^{-\alpha x} \chi_{[0, \infty]}$ and define,

$$
v(t):=(f * g)(t):=\int_{-\infty}^{t} f(x) e^{\alpha(x-t)} d x .
$$

Since $g \in L^{1}(\mathbb{R}), v \in P W_{\Omega}$. In the Fourier domain, $v$ and $f$ are related by

$$
\hat{f}(w)=(2 \pi i w+\alpha) \hat{v}(w) .
$$

In the time domain, this can be expressed as

$$
f(t)=\frac{\partial v(t)}{\partial t}+\alpha v(t) .
$$

Observation 1. The function $v$ is continuous and $v(t) \longrightarrow 0$, when $t \longrightarrow \pm \infty$.

Proof. We have already observed that $v \in P W_{\Omega}$. Since $\hat{v} \in L^{2}$ and $\operatorname{supp}(\hat{v}) \subseteq$ $[-\Omega, \Omega]$, we have that $\hat{v} \in L^{1}$ and the conclusion follows from the Riemann-Lebesgue Lemma.

The following straightforward equation relates $v$ to the integrate and fire process.

$$
\int_{s}^{t} f(x) e^{\alpha(x-t)} d x=v(t)-e^{\alpha(s-t)} v(s), \quad s \leq t .
$$

We can now prove that the output of the IF process is finite.

Proposition 1. Under Assumption 1, the following holds.

(a) The set of time instants produced by the integrate and fire scheme is a finite set $\left\{t_{0}, \ldots, t_{n}\right\}$.

(b) The numbers of time instants $t_{j}$ in a given finite interval $[a, b]$ is bounded by

$$
\frac{\|f\|_{2}}{\theta}(b-a)^{1 / 2}+1
$$

(c) If $f$ is integrable, the total number of time instants is bounded by

$$
\frac{\|f\|_{1}}{\theta}+1
$$

Proof. We first prove (b) and (c). Let $[a, b]$ be an interval and let $\left\{t_{j}, \ldots, t_{j+m-1}\right\}$ be $m$ consecutive time instants contained in $[a, b]$. If $m \leq 1$ the bound is trivial, so assume that $m \geq 2$. For each $0 \leq k \leq m-2$, using Equation (2) we have,

$$
\begin{aligned}
\theta & =\left|\int_{t_{j+k}}^{t_{j+k+1}} f(x) e^{\alpha\left(x-t_{j+k+1}\right)} d x\right| \\
& \leq \int_{t_{j+k}}^{t_{j+k+1}}|f(x)| d x .
\end{aligned}
$$

Summing over the $m-1$ intervals determined by the points $\left\{t_{j}, \ldots, t_{j+m-1}\right\}$ we have,

$$
(m-1) \theta \leq \int_{a}^{b}|f(x)| d x
$$


Letting $a=-\infty$ and $b=+\infty$ yields (b). For (a), Hölder's inequality gives,

$$
(m-1) \theta \leq\|f\|_{2}(b-a)^{1 / 2},
$$

and the conclusion follows.

Now we prove (a). Assume on the contrary that the IF process goes on forever producing an infinite set of instants $\left\{t_{j}: j \geq 0\right\}$. Given $s>t_{0}$, by part (b), only a finite number of instants $t_{j}$ belong to $\left[t_{0}, s\right]$. Therefore $t_{n} \rightarrow+\infty$, as $n \rightarrow+\infty$. Using Equations (7) and (2) it follows that,

$$
\begin{aligned}
\theta & =\left|\int_{t_{j}}^{t_{j+1}} f(x) e^{\alpha\left(x-t_{j+1}\right)} d x\right| \\
& =\left|v\left(t_{j+1}\right)-e^{\alpha\left(t_{j}-t_{j+1}\right)} v\left(t_{j}\right)\right| \leq\left|v\left(t_{j+1}\right)\right|+\left|v\left(t_{j}\right)\right| .
\end{aligned}
$$

This contradicts Observation 1

\section{The RECONSTRUCTION}

We now address the problem of approximately reconstructing a bandlimited function from the integrate and fire output. Since the samples are taken in the half-line $\left[t_{0},+\infty\right)$ we will make some assumption about the size of $f$ before the initial instant. Roughly speaking, the integrate and fire process would not have produced any sample in the interval $\left(-\infty, t_{0}\right]$.

Assumption 2. The function defined in Equation (4) satisfies,

$$
|v(t)| \leq \theta \text {, for all } t \leq t_{0} .
$$

Note that by Observation 1, any $t_{0} \ll 0$ satisfies this assumption. To approximately reconstruct $f$ we will first approximately reconstruct $v$ from the integrate and fire output and then derive information about $f$ by means of Equation (5). We will use the structure of the IF process to produce a number of approximate samples for $v$.

First we argue that, from the output of the IF process, we have enough information to approximate $v$ on the time instants $\left\{t_{0}, \ldots, t_{n}\right\}$. Rewriting Equation (3) in terms of $v$ (cf. Equation (7)) we have,

$$
v\left(t_{j+1}\right)=e^{\alpha\left(t_{j}-t_{j+1}\right)} v\left(t_{j}\right)+q_{j+1}, \quad(0 \leq j \leq n-1) .
$$

Since the value $v\left(t_{0}\right)$ may not be exactly known we cannot determine from this recurrence relation all the values $v\left(t_{j}\right)$. However, we can construct an approximation to these values. Let $w_{0}:=0$ and define recursively,

$$
w_{j+1}=e^{\alpha\left(t_{j}-t_{j+1}\right)} w_{j}+q_{j+1}, \quad(0 \leq j \leq n-1) .
$$

Observe that Assumption 2 implies that $\left|w_{0}-v\left(t_{0}\right)\right| \leq \theta$. Using this estimate as a starting point we can iterate on Equation (9) and (10) to get,

$$
\left|w_{j}-v\left(t_{j}\right)\right| \leq \theta, \quad(0 \leq j \leq n) .
$$

Consequently, using only the output of the IF sampling scheme, we have constructed a set of values $\left\{w_{0}, \ldots, w_{n}\right\}$ that approximates $v$ on the instants $\left\{t_{0}, \ldots, t_{n}\right\}$. The second step is to approximate $v$ on an arbitrary point of $\mathbb{R}$. 
6 H.G. FEICHTINGER, J. C. PRINCIPE, J.L. ROMERO, A. S. ALVARAdO, AND G. VELASCO

To this end observe that, according to the definition of $t_{j}$ as the minimum number satisfying Equation (2), we have that,

$$
\left|\int_{t_{j}}^{t} f(x) e^{\alpha(x-t)} d x\right| \leq \theta, \quad \text { for all } t \in\left[t_{j}, t_{j+1}\right] .
$$

Rewriting this inequality in terms of $v$ (cf. Equation (7)) gives,

$$
\left|v(t)-e^{\alpha\left(t_{j}-t\right)} v\left(t_{j}\right)\right| \leq \theta, \quad \text { for all } t \in\left[t_{j}, t_{j+1}\right] .
$$

Combining this last inequality with (11) yields,

$$
\left|v(t)-e^{\alpha\left(t_{j}-t\right)} w_{j}\right| \leq 2 \theta, \quad \text { for all } t \in\left[t_{j}, t_{j+1}\right] .
$$

We now show that this inequality allows us to approximate $v$ anywhere on the line.

Claim 1. Given an arbitrary time instant $t \in \mathbb{R}$, choose $x \in \mathbb{R}^{d}$ in the following way:

(a) if $t<t_{0}$, let $x:=0$,

(b) if $t$ belongs to some (unique) interval $\left[t_{j}, t_{j+1}\right)$, let $x:=e^{\alpha\left(t_{j}-t\right)} w_{j}$,

(c) if $t \geq t_{n}$, let $x:=e^{\alpha\left(t_{n}-t\right)} w_{n}$.

Then, $|v(t)-x| \leq 2 \theta$.

Remark 1. Observe that the procedure to obtain $x$ from $t$ depends only on the output of the IF process.

Proof. For case (a), the conclusion follows from Assumption 2 . For case (b), the conclusion follows from Inequality (13). For case (c), the fact that the fire condition is never satisfied after $t_{n}$ gives,

$$
\left|v(t)-e^{\alpha\left(t_{n}-t\right)} v\left(t_{n}\right)\right| \leq \theta .
$$

Combining this estimate with Inequality (11), the conclusion follows.

We will now choose a window function.

Assumption 3. A Schwartz class function $\psi$ such that

- $\hat{\psi} \equiv 1$ on $[-\Omega, \Omega]$, and,

- $\hat{\psi}$ is compactly supported,

has been chosen.

Since $v \in P W_{\Omega}$, the classic oversampling trick for bandlimited functions (see for example [6] or [7]) implies that there exists a number $0<\beta<(2 \Omega)^{-1}$ such that

$$
v=\sum_{k \in \mathbb{Z}} v(\beta k) \psi(\cdot-\beta k) .
$$

Using the procedure described in Claim 1, we produce a set $\left\{s_{k}\right\}_{k \in \mathbb{Z}}$ such that

$$
\left|v(\beta k)-s_{k}\right| \leq 2 \theta, \text { for all } k \in \mathbb{Z}
$$

Let $\varphi$ be the function defined by,

$$
\hat{\varphi}(w)=(2 \pi i w+\alpha) \hat{\psi}(w) .
$$


It follows that $\varphi$ is also a Schwartz function. Moreover, using Equation (5) we have that,

$$
f=\sum_{k \in \mathbb{Z}} v(\beta k) \varphi(\cdot-\beta k) .
$$

Observe that, since $v \in P W_{\Omega}$, the sequence $\{v(\beta k)\}_{k} \in \ell^{2}$ and the series in Equation (18) converges in $L^{2}$ and uniformly - in fact, it converges in the Wiener amalgam norm $W\left(C_{0}, L^{2}\right)$, see for example [6], [7] and [2].)

Now we can define the approximation of $f$ constructed from the IF samples. Let,

$$
\tilde{f}:=\sum_{k \in \mathbb{Z}} s_{k} \varphi(\cdot-\beta k) .
$$

Since, by Inequality (16), the sequence $\left\{s_{k}\right\}_{k}$ is bounded and $\varphi$ is a Schwartz function, it follows that Equation (19) defines a bounded function and that the convergence is uniform (see [6] or [2].)

The reconstruction algorithm consists then of calculating the approximated samples $\left\{s_{k}\right\}_{k}$ following Claim 1 and then convolving them with the kernel $\varphi$, that can be pre-calculated.

We now give a precise error bound for the reconstruction.

Theorem 1. Under Assumptions 1, 2 and 3, the function defined by Equation (19) satisfies,

$$
\|f-\tilde{f}\|_{\infty} \leq C \theta
$$

for some constant $C$ that only depends on $\Omega$ and the window function chosen in Assumption 3 .

Proof. According to Equations (18), (19) and Inequality (16),

$$
\begin{aligned}
\|f-\tilde{f}\|_{\infty} & \leq \operatorname{supess} \sum_{k \in \mathbb{Z}}\left|v(\beta k)-s_{k}\right||\varphi(\cdot-\beta k)| \\
& \leq 2 \theta \text { supess } \sum_{k \in \mathbb{Z}}|\varphi(\cdot-\beta k)| .
\end{aligned}
$$

It suffices to define $C:=2 \sup \sum_{k \in \mathbb{Z}}|\varphi(\cdot-\beta k)|$. Since $\varphi$ is a Schwartz function, $C<+\infty$ (see for example [5]).

Remark 2. We currently do not know what choice of the window function $\psi$ minimizes the constant in the theorem. A more detailed study of the choice of the window function should not only consider the size of that constant but also the rate of convergence of the series in Equation (19).

\section{Numerical experiments}

We study the behavior of the reconstruction algorithm under variations in the threshold and the oversampling period for a specific choice of reconstruction kernel $\psi$. The test signal $f$ is of finite length and real valued, produced as a linear combination of five 'sinc' kernels $(\sin (\pi x) /(\pi x))$ at a $1 \mathrm{~Hz}$ frequency, with random locations and weights. The amplitude of the input has been normalized to 1. Although the theory covers infinite dimensional spaces, our simulations are limited by the practical implementations of the sampler and algorithms. The effects of truncation and quantization are not considered here. 
This signal is encoded by the IF sampler with $\alpha=1$ and recovered using the procedure described in Section 4 The reconstruction kernel $\psi$ is a raised cosine, defined by,

$$
\psi(t)=\operatorname{sinc}\left(t / T_{s}\right) \cos \left(\pi \gamma t / T_{s}\right)\left(1-\frac{4 \gamma^{2} t^{2}}{T_{s}^{2}}\right)^{-1},
$$

where $\gamma=0.5$ and $T_{s}=0.25$ are determined by the maximum input frequency $\Omega$ and the desired oversampling period $\beta$ (cf. Equation (15).) Figure 2(a) shows the raised cosine $\psi$ in the time and frequency domain. Observe that the spectrum of $\psi$ is constant for frequencies less than the input bandwidth and then decays smoothly towards zero. The corresponding kernel $\varphi$ (cf. Equation (17)) is shown in Figure 2(b) Using $\varphi$ we recover $\tilde{f}$ (cf. Equation (19).), an approximation of $f$
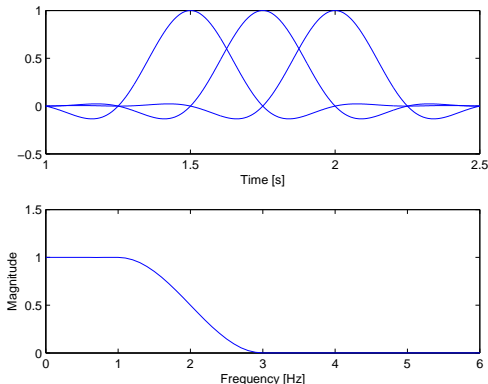

(a) Kernel $\psi$
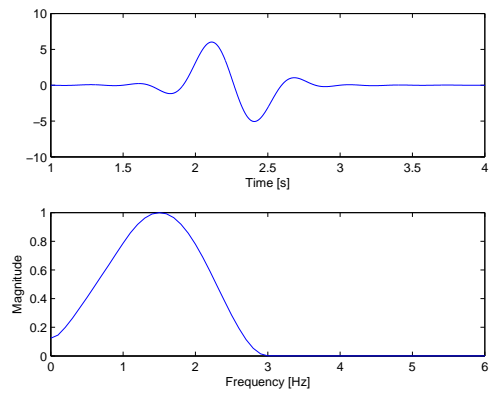

(b) Kernel $\varphi$.

Figure 2. Reconstruction kernels.

as shown in Figure 3 As expected the error decreases in regions with high density of samples. This behavior is evident from Figure 4 the dense regions imply that the uniform samples will most likely coincide with the estimated values of $v(t)$ at the sample locations. On the other hand, for samples that are far apart the approximation follows a exponential decay from its original value which is not the natural trend in the signal. Figure 4 shows $v(t)$ (solid line), and the approximated samples of $v$ on the lattice $\beta \mathbb{Z}$, constructed using the procedure described in Claim 1 called $\left\{s_{k}\right\}_{k}$ and the envelope $v(t) \pm \theta$ where these samples are known to lie (dashed line.)

Currently the reconstruction algorithm uses the approximated samples of $v(t)$ at the pulse locations to define the piecewise exponential bound and estimate the reconstruction coefficients on the uniform lattice. Based on the numerical experiments the algorithm can be improved by including the estimated value of $v(t)$ at the pulse locations although it implies reconstruction on a nonuniform grid. For both cases similar error bounds can be defined as in Theorem 1. The variation of the error in relation to the threshold (pulse rate) is shown in Figure 5 . The error depends on the choice of generator and the oversampling period $\beta$, as seen in Figure 6. The relationship between the kernels and the optimal oversampling period is still not evident. 


\section{Acknowledgements}

The second and fourth authors were supported by NINDS (Grant Number: NS053561). The third author was partially supported by the following grants: PICT06-00177, CONICET PIP 112-200801-00398 and UBACyT X149. The third and fourth authors' visit to the Numerical Harmonic Analysis Group (NuHAG) of the University of Vienna was funded by the European Marie Curie Excellence Grant EUCETIFA FP6-517154.

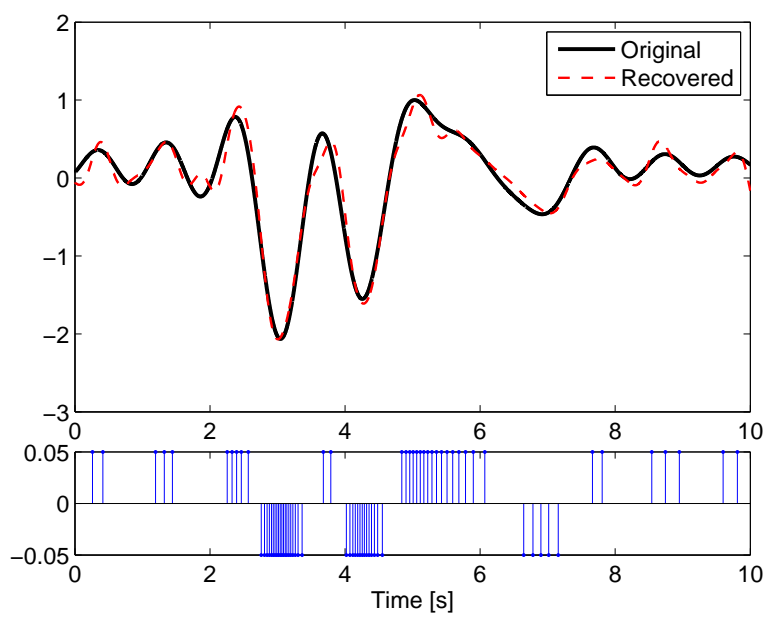

Figure 3. Reconstruction of $f(t)$ from the impulse train.

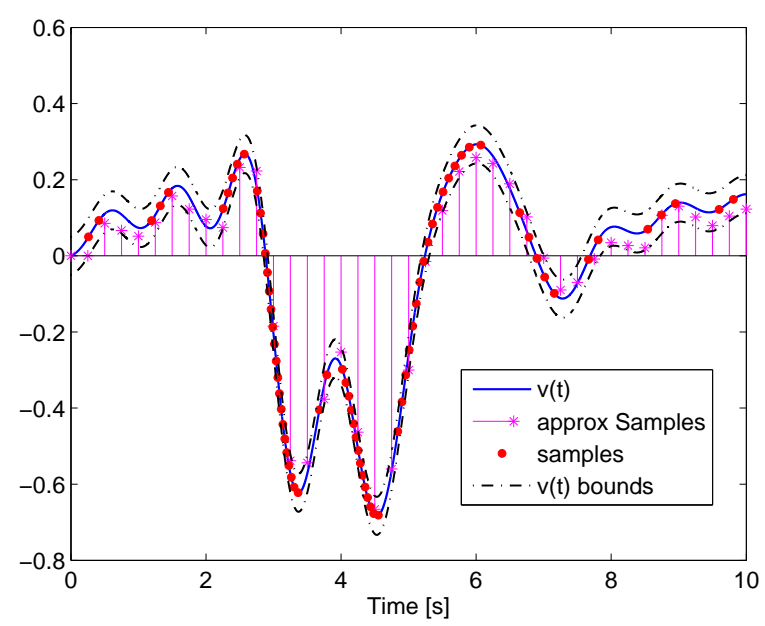

FiguRE 4. Reconstruction of $v(t)$ with $\beta=1 / 4, \theta=0.05$ 


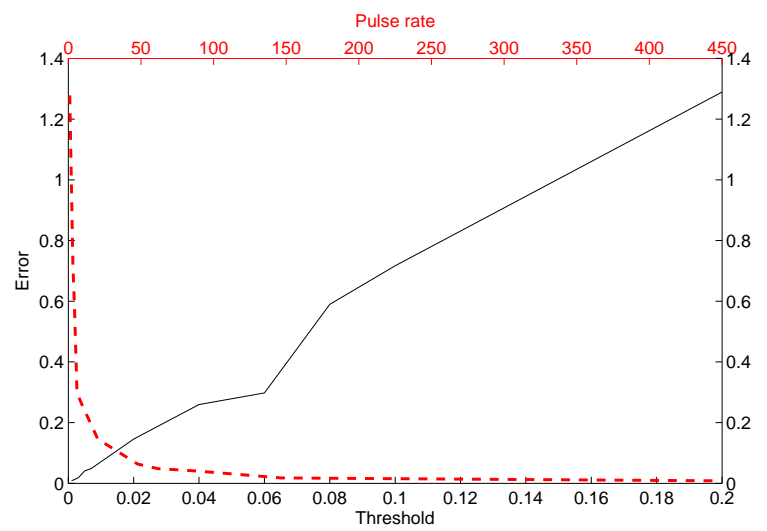

FiguRE 5 . Variation of the error $\|f-\widehat{f}\|_{\infty}$ in relation to the threshold and pulse rate (dotted line) with $\beta=0.25$.

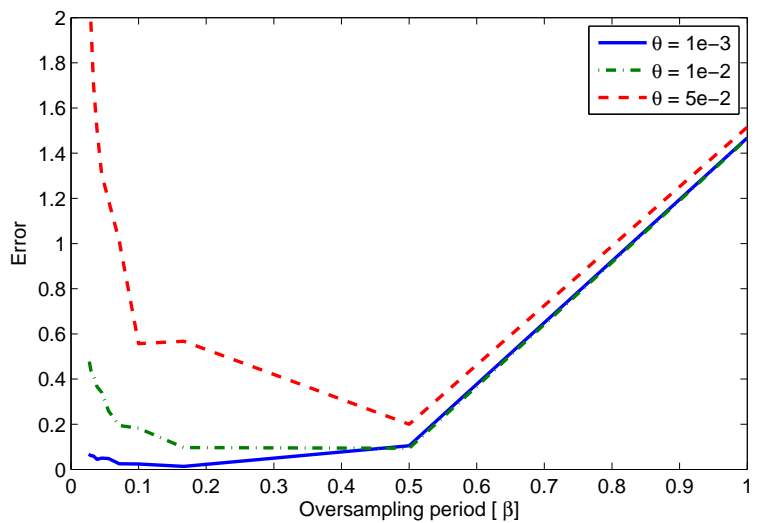

FiguRE 6. Variation of the error in relation to the oversampling period for different thresholds. The error is defined as $\|f-\widehat{f}\|_{\infty}$. 


\section{REFERENCES}

[1] A. Aldroubi. Non-uniform weighted average sampling and reconstruction in shift-invariant and wavelet spaces. Appl. Comput. Harmon. Anal., 13(2):151-161, 2002.

[2] A. Aldroubi and K. Gröchenig. Nonuniform sampling and reconstruction in shift-invariant spaces. SIAM Rev., 43(4):585-620, 2001.

[3] A. Alvarado, J. Principe, and J. Harris. Stimulus reconstruction from the biphasic integrateand-fire sampler. In Neural Engineering, 2009. NER '09. 4th International IEEE/EMBS Conference on, pages 415-418, April 29 2009-May 22009.

[4] D. Chen, Y. Li, D. Xu, J. Harris, and J. Principe. Asynchronous biphasic pulse signal coding and its cmos realization. Circuits and Systems, 2006. ISCAS 2006. Proceedings. 2006 IEEE International Symposium on, pages 4 pp.-2296, 0-0 2006.

[5] H. G. Feichtinger. Banach convolution algebras of Wiener type. In B. Sz. Nagy and J. Szabados, editors, Proc. Conf. on Functions, Series, Operators, Budapest 1980, volume 35 of Colloq. Math. Soc. Janos Bolyai, pages 509-524, Amsterdam, 1983. North-Holland.

[6] H. G. Feichtinger. Wiener amalgams over Euclidean spaces and some of their applications. In K. Jarosz, editor, Function Spaces, Proc Conf, Edwardsville/IL (USA) 1990, volume 136 of Lect. Notes Pure Appl. Math., pages 123-137, New York, 1992. Marcel Dekker.

[7] H. G. Feichtinger and K. Gröchenig. Theory and practice of irregular sampling. In J. Benedetto and M. Frazier, editors, Wavelets: Mathematics and Applications, Studies in Advanced Mathematics, pages 305-363, Boca Raton, FL, 1994. CRC Press.

[8] W. Gerstner and W. Kistler. Spiking neuron models. Cambridge University Press, 2002.

[9] A. A. Lazar and E. A. Pnevmatikakis. Reconstruction of sensory stimuli encoded with integrate-and-fire neurons with random thresholds. EURASIP Journal on Advances in Signal Processing, 2009, July 2009.

[10] A. A. Lazar, E. K. Simonyi, and L. T. Toth. A Toeplitz formulation of a real-time algorithm for time decoding machines. In Proceedings of the Conference on Telecommunication Systems, Modeling and Analysis, November 2005.

[11] A. A. Lazar and L. T. Toth. Time encoding and perfect recovery of bandlimited signals. In IEEE International Conference on Acoustics, Speech and Signal Processing, volume 6, pages VI709-712, April 2003.

[12] F. Rieke, D. Warland, de Ruyter, and W. Bialek. Spikes: Exploring the Neural Code. The MIT Press, 1997.

[13] J. C. Sanchez, J. C. Principe, T. Nishida, R. Bashirullah, J. G. Harris, and J. A. B. Fortes. Technology and signal processing for brain-machine interfaces. Signal Processing Magazine, IEEE, 25(1):29-40, 2008.

[14] H. Schwab. Reconstruction from Averages. PhD thesis, Dept. Mathematics, Univ. Vienna, 2003.

[15] W. Sun and X. Zhou. Reconstruction of band-limited functions from local averages. Constr. Approx., 18(2):205-222, 2002.

[16] D. Wei. Time based analog to digital converters. PhD thesis, University of Florida, 2005. 
12H.G. FEICHTINGER, J. C. PRINCIPE, J.L. ROMERO, A. S. AlvARAdO, AND G. VELASCO

Faculty of Mathematics, University of Vienna, Nordbergstrasse 15, Vienna, Austria

E-mail address, Hans G. Feichtinger: hans.feichtinger@univie.ac.at

Department of Electrical and Computer Engineering, University of Florida, Gainesville, FL 32611, USA

E-mail address, J. C. Príncipe: principe@cnel.ufl.edu

Departamento de Matemática, Facultad de Ciencias Exactas y Naturales, Universidad de Buenos Aires, Ciudad Universitaria, Pabellón I, 1428 Capital Federal, ArGentina, AND CONICET, ARgentina

E-mail address, José Luis Romero: jlromero@dm.uba.ar

Department of Electrical and Computer Engineering, University of Florida, Gainesville, FL 32611, USA

E-mail address, Alexander Singh Alvarado: asingh@cnel.ufl.edu

Faculty of Mathematics, University of Vienna, Nordbergstrasse 15, Vienna, Austria, and Institute of Mathematics, University of the Philippines Diliman, Quezon City, PHILIPPINES

E-mail address, Gino Angelo Velasco: gino.velasco@univie.ac.at 